\title{
Research on Hardware-in-the-loop Simulation of Continuous Wave Doppler Radio Fuze
}

\author{
Fengchen $\mathrm{Li}^{\text {a }}$, Dongguang Zuo, Longtao $\mathrm{Hu}^{\mathrm{b}}$ \\ Xi'an Research Institute of High Technology, Xi 'an 710025, China. \\ a610580194@qq.com, b523098802@qq.com
}

\begin{abstract}
Based on the action principle of continuous wave Doppler Radio fuze, the hardware-in-the-loop simulation system is designed. Will test the role of the fuze Doppler signal which the fuze generated will be collected by using precision operational amplifier OPA378, after its adjustment to the processor, the processor will collect the sig nal to AD conversion, and upload to the host computer, the received dopple signal waveform is displayed, to stop after the threshold accepted. Finally, the experimental results show that the system can simulate the CW Doppler airfuse fuze.
\end{abstract}

Keywords: Radio Fuze; HILS; Continuous Wave; Doppler Signal.

\section{Introduction}

The main role of fuze is to detonate ammunition, it plays an important part of the warhead. Fuze development is a very complex process, and the development of the main work is concentrated in the trial and test process, there are many methods [1] of the fuze comprehensive performance evaluation, Traditional shooting test show more realistic assessment of fuze performance, But a long cycle, not economic and other shortcomings exist, and taking use of HILS technology for testing fuze is a viable method.

Known as radar fuze because the working principle of radio fuze and radar similar, is a kind of active fuze, the Doppler Effect is an important means of radio fuze to recognite target, so the fuze is also known as continuous wave doppler radio fuze [2]. When the relative movement of the projectile, the echo frequency will change, which act as a basis for research and design of HILS system [3].

\section{Overall Design}

Hardware-in-the-loop simulation device mainly includes host computer, adapter, test fuze. Host computer is the control computer of the hardware-in-the-loop simulation platform, the adapter establishes the data transmission connection between the host computer and the fuze. The overall block diagram shown in Figure 1.

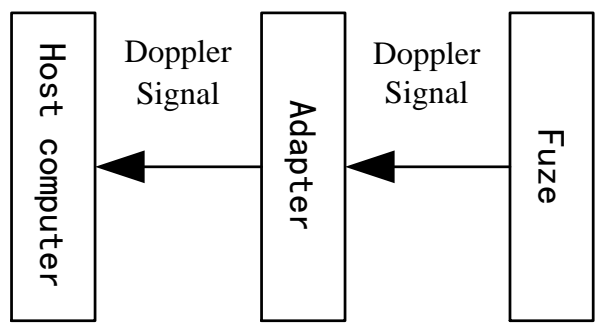

Fig. 1 Overall Structure Diagram

When the hardware-in-the-loop simulation is carried out, the external push plate moves relative to the fuze. The fuze produces the Doppler signal, which is collected by the adapter and uploaded to the host computer.

\section{Adapter Design}

The adapter is connected to the host computer through the RS232 to USB interface. The power supply is a rechargeable battery and provides $24 \mathrm{~V}$ DC power for the fuze. At the same time as the input voltage of the regulator module. The internal signal acquisition board of the adapter collects the 
Doppler signals generated by the test fuses and uploads them to the host computer. The signal acquisition board mainly includes a voltage regulation module and a signal acquisition module.. The structure shown in Figure 2:

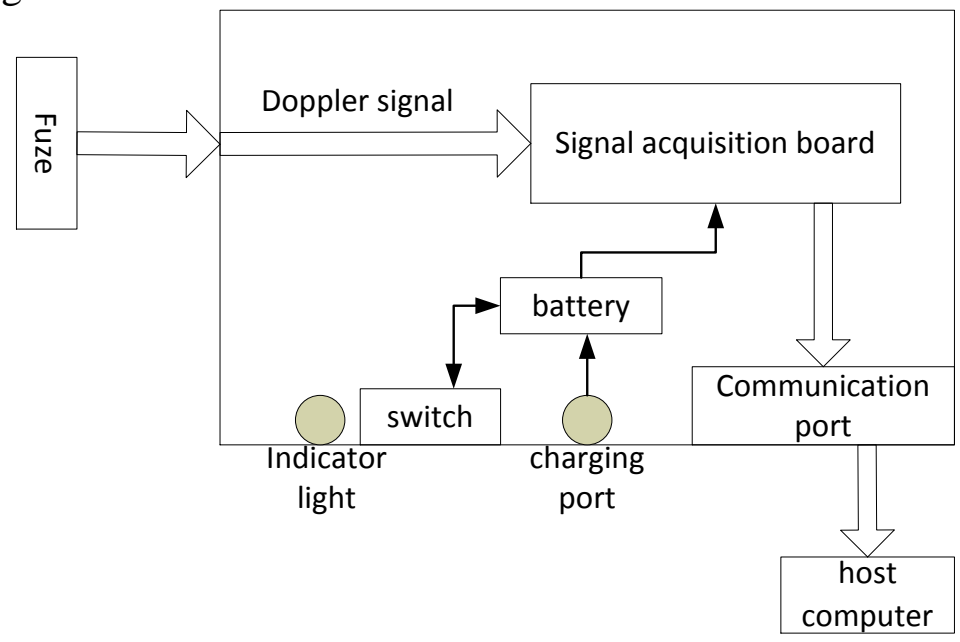

\subsection{Signal Acquisition Module}

Fig. 2 Adapter block diagram

STM32F303 is used as the processor, the chip uses the Cortex-M4 core, the maximum clock frequency is $72 \mathrm{MHz}$, on-chip $256 \mathrm{~KB}$ FLASH, 48KB RAM, the processor internal reset and power-down detection function has been relatively complete, So in the minimum system design, reset circuit using a relatively simple RC power-on circuit. Processor through the communication port connected with the host computer, the schematic diagram shown in Figure 3:

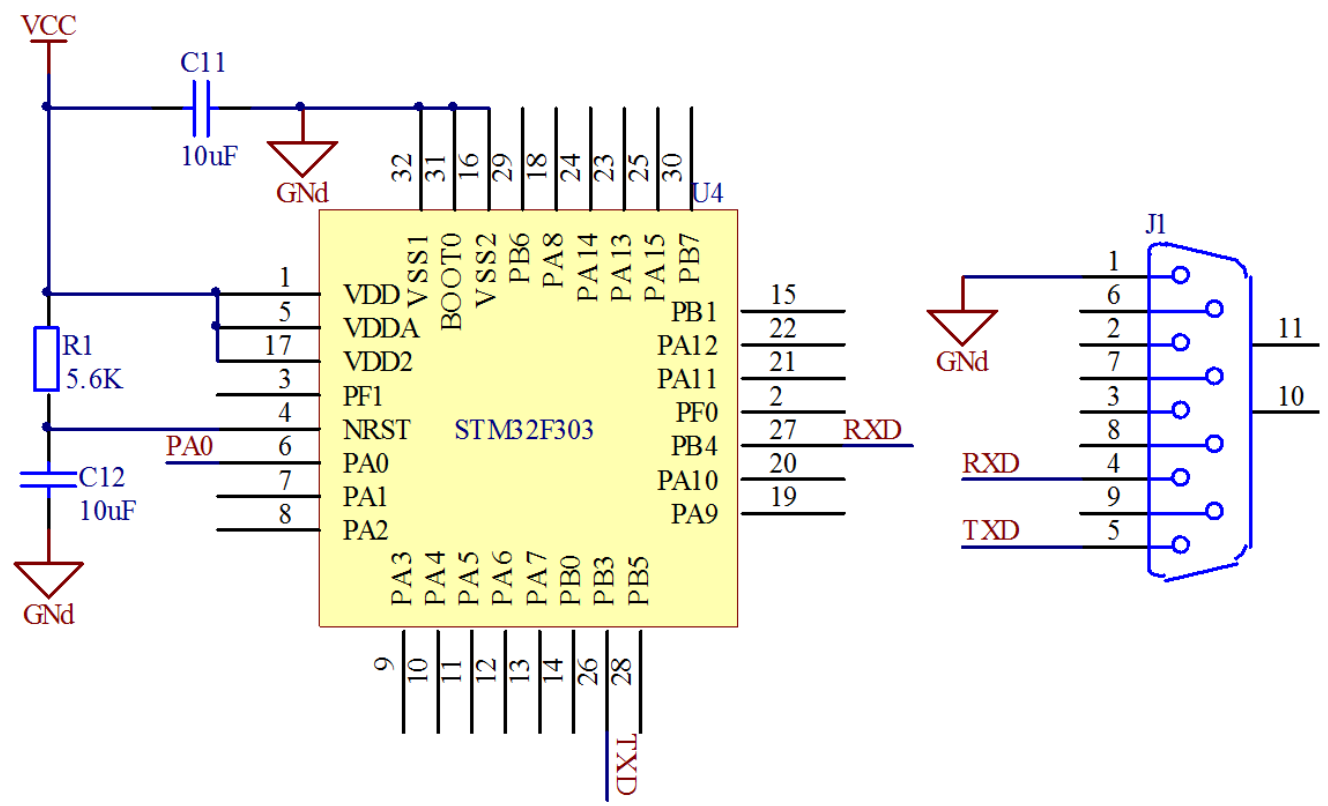

Fig. 3 Minimum System and Communication Interface

The main function [4] of the processor is to collect signals and upload to the host computer, after the internal initialization of the system power, receive the output signal from the OPA378, A/D converted upload to the host computer, and stop working after power down, The flow chart shown in Figure 4: 


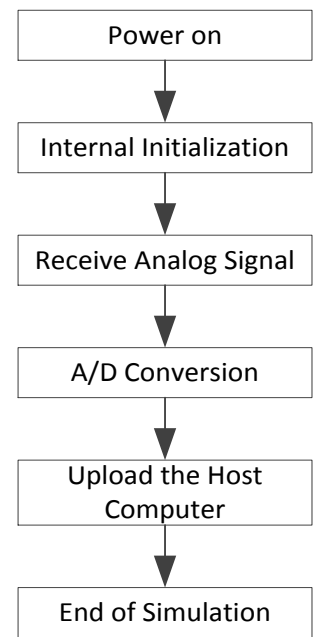

Fig. 4 Software flow chart

Doppler signal from fuze transmitted to precision op amp OPA378, the supply voltage of $2.2 \mathrm{~V}-5.5 \mathrm{~V}$, with low noise, zero drift, low quiescent current characteristics, it also has EMI filtering and other functions, the schematic diagram 5 shows:

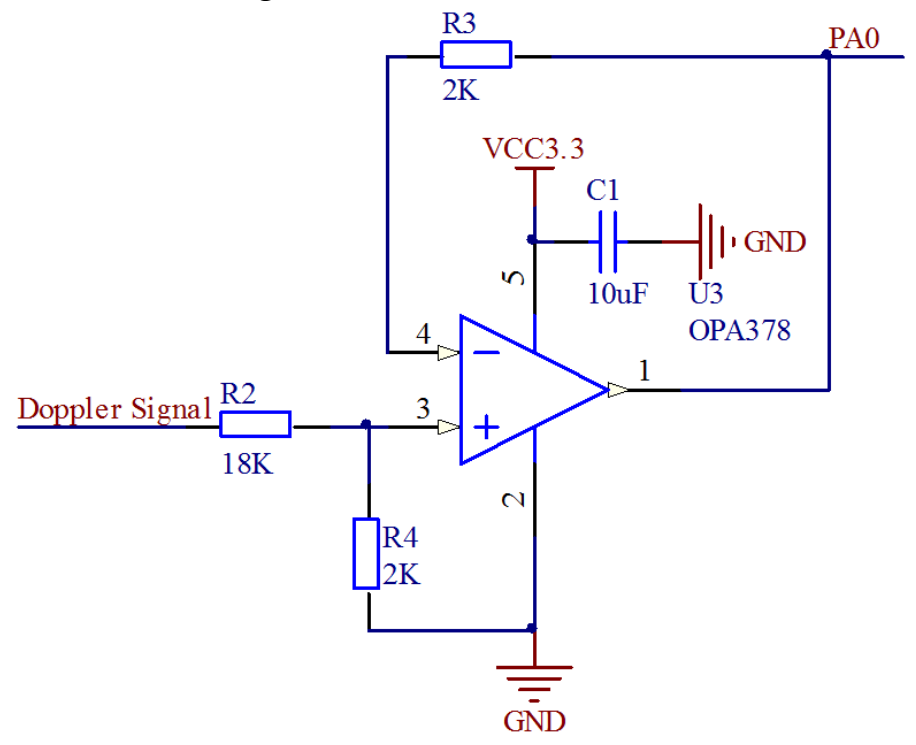

Fig. 5 Signal Aquisition Circuit

\subsection{Regulator modular}

According to the power supply requirements, the regulator module is designed [5], which includes $\mathrm{DC} / \mathrm{DC}$ switching power, $\mathrm{DC} / \mathrm{DC}$ isolation power, and regulator circuit.

(1) DC/DC switching power:

LM2596 switching voltage regulator is used in switching power [6], the regulator is a newly developed miniaturized power switch module, which take use of microelectronics technology, the small surface mount integrated circuit and micro-electronic components assembled into one component. With a wide input voltage range, the smaller the output voltage ripple, high conversion efficiency. Its product features:

Maximum input voltage: $40 \mathrm{~V}$;

Maximum output current: $3 \mathrm{~A}$;

Voltage conversion efficiency: $85 \%$;

Switching power convert the $24 \mathrm{~V}$ DC to $5 \mathrm{~V}$, in order to ensure normal operation of the other parts of the regulator module is stable, the following design of the switching power is:

Input protection

The diode is a very effective protection device that can be changed in a very short time when the high energy of the transient impinges on its two stages in a short period of time. Its two-stage impedance, from high impedance to low impedance, absorb most of the surge power, thus protecting 
the electronic circuit. The use of bidirectional transient suppression diodes, regardless of the circuit by the forward or reverse impact, can effectively play a protective role [7].

Anti-interference design

The voltage conversion process need to filter [8], in the LM2596 input and output were connected to a maximum input voltage of 1.5 times the electrolytic capacitor, the input capacitor can prevent a large transient voltage, while LM2596 work Room to provide transient current; the output capacitor from the main role of filtering to improve stability. Diode D2 mainly plays the role of freewheeling [9].

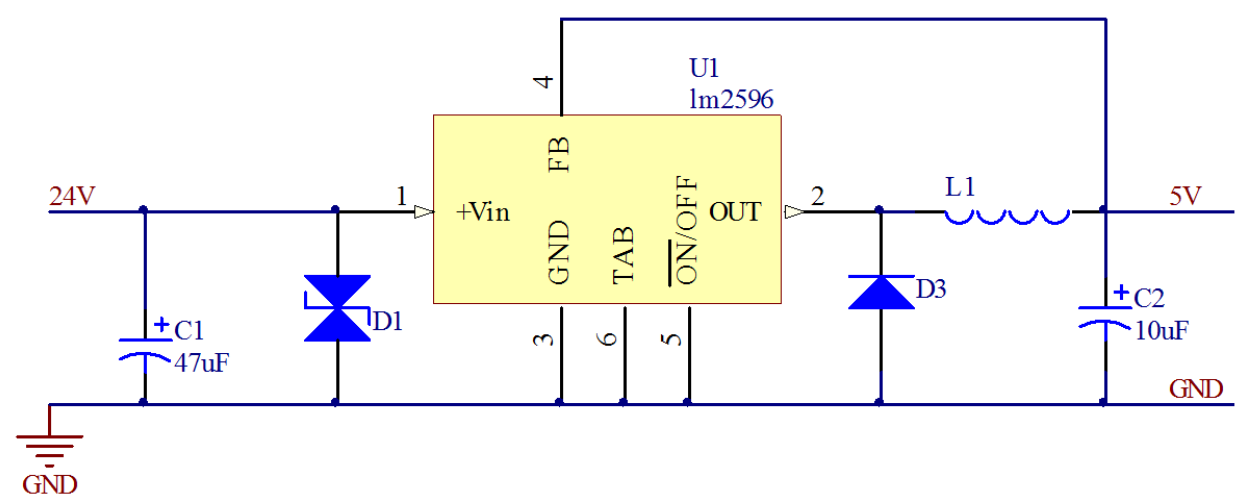

(2) DC / DC isolated power:

Fig. 6 Switching Power Supply

In order to eliminate the interference between the test fuze and the signal acquisition module, the DCP020507 is chosen as the isolated power supply. The chip is one kind of switching power supply, its most important characteristic is that the output voltage and the input voltage are not common, The isolation. Its product features:

Input voltage: $4.5 \mathrm{~V} \sim 5.5 \mathrm{~V}$;

Maximum output current: $100 \mathrm{~mA}$;

Voltage conversion efficiency: $71 \%$;

Switching power supply 5V DC voltage generated by the DCP020507 isolation, the input and output side of the capacitor is mainly from the role of stabilizing voltage, the schematic shown in Figure 6:

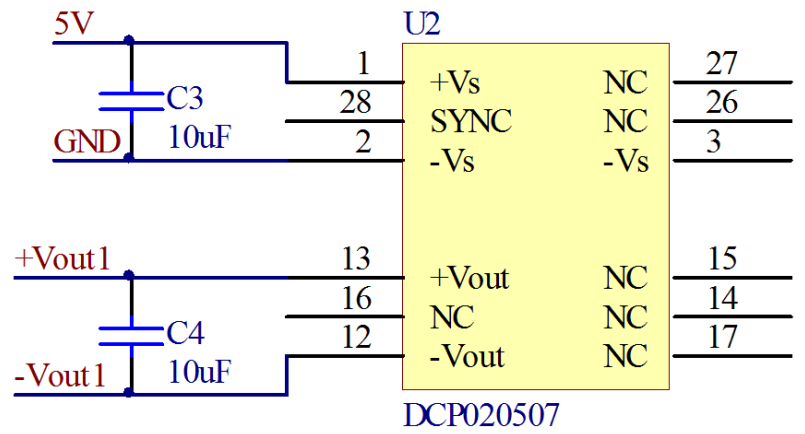

(3) Regulator circuit

Fig. 7 Isolation Module

Voltage regulator circuit part of the main need to stabilize the voltage regulator $3.3 \mathrm{~V}$, use low-dropout linear regulator LM1117, the regulator chip has a stable output, low quiescent current, external components and so on. Its product features:

Maximum input voltage: $20 \mathrm{~V}$

Maximum output current: $800 \mathrm{~mA}$

Quiescent current: 75uA

Regulator module schematic as shown, mainly using LM1117-3.3 fixed output type, in order to improve transient response and stability of the chip input, the output of each access to a $10 \mathrm{uF}$ capacitor, as shown in Figure 8: 


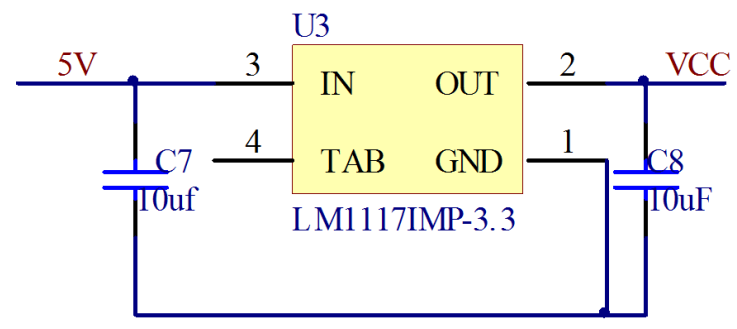

Fig. 8 Regulator Modular

\section{PC Software Design and Test Results}

PC interface from top to bottom, including two parts: the menu bar, telemetry data area.

The menu bar includes the "Communication Ports" menu, the "Start" menu, the "Stop" menu, and the "Exit" menu. The menu item under the "Communication Ports" menu is used to set the communication port. The "Start" menu is used to start the simulation process. After clicking the "Start" menu, the simulation software releases the start simulation command and starts to collect telemetry data from the fuze. The acquired telemetry data is displayed in the telemetry data area in the simulation software interface. The "Stop" menu is used to stop the simulation process. The "Exit" menu is used to exit the simulation software.

The telemetry data area is used to display the telemetry data from the simulated fuzes. Blank when telemetry data is not displayed. When the start-up simulation is turned on, this area displays the telemetry data from the simulated fuzes, as shown in Figure 9:

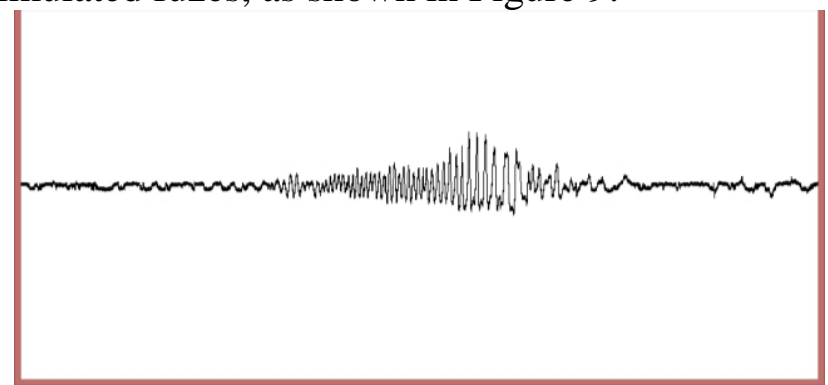

Fig. 9 Test Results

\section{Summary}

In this paper, the design of HILS system for continuous wave doppler fuze is introduced in detail. Firstly, the HILS system is designed according to the working principle of the fuze. Then the design of the adapter and host computer is introduced in detail., The final test results show that the system can work properly, which of great significance in the development of continuous wave doppler fuze. The HILS of the fuze is a long process. In this paper, the HILS is designed for a kind of fuze and the other fuze such as the penetration fuze and the trigger fuze cannot be simulated. We need to study the generalization of the hardware-in-the-loop simulation system and the accuracy of the simulation model in detail.

\section{References}

[1] Zhang Zhian. Study on the Hardware in the Loop Simulation Technology for Hard Target Penetration Fuze [D]. Nanjing University of Science and Technology, 2007.

[2] Yuan Kun, Qu Jiahui. Simulation of Continuous Wave Doppler Radio Fuze and its Signal Processing [J]. Journal of Shenyang Ligong University, 2013(01):37-41.

[3] Wang Lei. Study on the Hardware in the Loop Simulation System for Hard Target Penetration Fuze. [D]. Nanjing University of Science and Technology, 2013. 
[4] Cheng Liangliang, Zhang Zhongxiang, Chen Jiabao, etc. Design of Multichannel Wireless Synchronous Plasma Force Acquisition System Based on STM32 [J]. Journal of Anqing Teachers College (Natural Science Edition), 2015(04):69-72.

[5] Zhang Shutuan, Shi Xianjun, Xiao Zhicai. Design and Implementation of Multi-output DC Power Module [J]. Marine Electric \& Electronic Technology, 2011 (11) :62-64.

[6] Lv Changzhi, Ma Weidong, Xie Xuesong, etc. Research on the reliability of DC/DC power module [J]. Journal of Beijing University of Technology, 2010(07):890-895.

[7] LIANG Yu, WANG Shezhu. The discussion of the loss resistance about inductor and capacitor [J]. Physics Examination and Testing, 2002(5): 17-19.

[8] Gou Huilan, Liu Guangchao. Minimum System and Serial Communication Implementation Based on STM32 [J]. Industrial Control Computer, 2012(09):26-28.

[9] LM2596. Datasheet. 